CLINICAL STUDY

\title{
Serum concentrations of atherogenic proteins neutrophil gelatinase-associated lipocalin and its complex with matrix metalloproteinase-9 are significantly lower in women with polycystic ovary syndrome: hint of a protective mechanism?
}

\author{
Evanthia Diamanti-Kandarakis, Sarantis Livadas, Stylianos A Kandarakis, Alexandra Margeli ${ }^{1}$ \\ and Ioannis Papassotiriou ${ }^{1}$ \\ Endocrine Section, First Department of Medicine, Medical School, Laiko General Hospital, University of Athens, 11527 Athens, Greece and ${ }^{1}$ Department of \\ Clinical Biochemistry, 'Aghia Sophia' Children's Hospital, 11527 Athens, Greece \\ (Correspondence should be addressed to I Papassotiriou; Email: biochem@paidon-agiasofia.gr)
}

\begin{abstract}
Background: Neutrophil gelatinase-associated lipocalin (NGAL) and matrix metalloproteinase-9 (MMP9) have been considered as important mediators of vascular remodeling and plaque instability. The formation of a complex with NGAL and MMP-9 is crucial for atherotic plaque erosion and thrombus formation. In women with polycystic ovary syndrome (PCOS), the incidence of cardiovascular clinical events is not increased, despite the fact that they display a wide spectrum of risk factors. Since the instability of atherosclerotic plaque is a key factor in the clinical manifestations of cardiovascular disease, molecules challenging the plaque stability should be investigated.

Aim: To determine serum levels of NGAL and MMP-9/NGAL complex in women with PCOS.

Subjects and methods: PCOS subjects (40) were compared with those (40) matched for age and body mass index (BMI) controls. In each subject, fasting levels of glucose, insulin, gonadotropins, estradiol, androgens, C-reactive protein, NGAL, and MMP-9/NGAL were determined.

Results: NGAL and MMP-9/NGAL complex levels were significantly lower in the PCOS group compared with controls $(30.4 \pm 24.3$ vs $70.7 \pm 37.9 \mu \mathrm{g} / \mathrm{l}, P<0.0001)$ and $(31.5 \pm 26.6$ vs $115.1 \pm 66.9 \mu \mathrm{g} / \mathrm{l}$, $P<0.0001)$ respectively. When patients and controls were stratified according to BMI, it was shown that NGAL and MMP-9/NGAL levels were significantly lower in lean $(P<0.0002$ and $P<0.0001$ respectively) and overweight $(P<0.0004$ and $P<0.002$ respectively) PCOS subjects compared with controls.

Conclusions: These findings indicate that NGAL and MMP-9/NGAL complex, two molecules that activate atherotic plaque erosion, is in lower concentrations in PCOS subjects. The role of NGAL and MMP-9/NGAL complex needs to be further investigated, since suppression of these atheromatous molecules might have a protective role in women with PCOS.
\end{abstract}

European Journal of Endocrinology 158 525-531

\section{Introduction}

Polycystic ovarian syndrome (PCOS) constitutes the most common endocrinopathy of women of reproductive age and its prevalence in the female population worldwide ranges from 6 to $7 \%$ (1). Elegant studies have revealed that women suffering from PCOS exhibit a broad spectrum of metabolic abnormalities, predisposing them to increased cardiovascular risk such as insulin resistance, dyslipidemia, fibrinolytic aberrations, subclinical inflammation, and raised levels of markers of oxidative stress $(2,3)$. Moreover, studies of endothelial function in this group of patients have disclosed the initiation of atherosclerotic process as evidenced by elevated levels of vasoconstrictive peptides, increased intima-media thickness of carotid arteries, disturbed endothelium-dependent flow-mediated dilatation of the arteries and increased coronary and aortic calcifications (4-6). Taking into account that these abnormalities are encountered as early as the second decade of life in patients with PCOS (7), these subjects represent significantly higher risk to develop cardiovascular incidents, in comparison with their age- and BMImatched peers.

Recent studies have shown that physical disruption of atherosclerotic plaque triggers thrombus formation, which may lead to myocardial infarction. In particular, gelatinase B, also known as matrix metalloproteinase-9 (MMP-9), an endopeptidase capable of degrading the molecular components of the extracellular matrix is 
thought to be associated with diseases such as abdominal aortic aneurysm, atherosclerosis, and plaque rupture $(8,9)$. Therefore, MMP-9 is considered to be an important mediator of vascular remodeling and plaque instability (10). MMP-9 action is enhanced by neutrophil gelatinase-associated lipocalin, also known as lipocalin-2 (NGAL), a $25 \mathrm{kDa}$ glycoprotein with many diverse functions, such as scavenger of bacterial products, modulator of inflammation, and apoptosis (11). The formation of a complex with NGAL and MMP9 is crucial for atherotic plaque erosion and thrombus formation (12-14). Hemdahl et al. have shown increased expression of NGAL and co-localization with MMP-9 in atherosclerotic plaques and myocardial infarction lesions (15).

A highly atherogenic vascular environment has been recognized in women suffering from PCOS (16). However, this atherogenic milieu is not conveyed to cardiovascular incidents, evident from the available studies and every day clinical practice (17). This fact implies the presence of potential protective mechanisms in PCOS women. Since MMP-9 and NGAL are two important factors affecting plaque stability leading to the development of cardiovascular incidents, the study of these molecules appears to be justified in women with PCOS.

\section{Subjects and methods}

\section{Subjects}

Women with PCOS, to participate in the study, had to fulfill the strict National Institute of Health (NIH) criteria (chronic anovulation - six or fewer menstrual cycles per year in association with hyperandrogenemia) (18). Hyperandrogenemia was assessed as total testosterone levels above the 95th percentile of the levels detected in the group of normal menstruating women. Other androgen excess disorders (congenital adrenal hyperplasia) were excluded accordingly. A synacthen test was conducted in each woman with a basal 17-hydroxyprogesterone (17-OHP) plasma level $>1.5 \mathrm{ng} / \mathrm{ml}$. Furthermore, all subjects in the control arm had regular periods and no hirsutism/acne. Exclusion criteria for the study included age over 40 years, known cardiovascular disease, thyroid disease, neoplasms, current smoking, diabetes mellitus, hypertension (blood pressure $>140 / 90 \mathrm{mmHg}$ ), renal impairment (serum creatinine $>120 \mu \mathrm{mol} / \mathrm{l}$ ). The enrolled population was clinically healthy and not suffering from any known chronic or acute disease. Oral contraceptives or other drugs that could interfere with the hormonal and metabolic studies, if administrated, were discontinued for at least 3 months before the study. The homeostasis assessment model (HOMA) assessed according to the formula, HOMA-IR = fasting insulin
$(\mathrm{mIU} / \mathrm{ml}) \times$ fasting glucose $(\mathrm{mmol} / \mathrm{l}) / 22.5$, has been used as a marker of insulin resistance (19).

The protocol was approved from the Institutional Review Committee of the 'Laiko' General University Hospital, and written informed consent was obtained from each subject before participation into the study.

\section{Assays}

The samples of control and PCOS subjects were collected at the same period of time between 0800 and $1000 \mathrm{~h}$, after an overnight fast. All samples were obtained during the early follicular phase (day 2-4 from the first day of spontaneous bleeding episode) or any time in an anovulatory phase with progesterone levels $<5 \mathrm{ng} / \mathrm{ml}$. In this study, we used a serum separator tube and allowed samples to clot for 30 min before centrifugation for $15 \mathrm{~min}$ at $\sim 1000 \boldsymbol{g}$. Serum was immediately stored at $-20{ }^{\circ} \mathrm{C}$ until assayed for glucose (GLU), lipids, insulin, total testosterone, sex hormone-binding globulin (SHBG), free testosterone, androstenedione $(\Delta 4 \mathrm{~A})$, luteinizing hormone (LH), follicle-stimulating hormone (FSH), 17-OHP, DHEAS, estradiol $\left(\mathrm{E}_{2}\right)$, high-sensitivity CRP (hsCRP), NGAL and MMP-9/NGAL; repeated freeze-thaw cycles were avoided.

All measurements were performed using the ChemWell Analyzer (Awareness Technology, Palm City, FL, USA), unless otherwise stated. Synacthen test was performed using Synacthen $0.25 \mathrm{mg} / \mathrm{ml}$ (Novartis Pharma SA, Rueil-Malmaison, France). Plasma fasting glucose $(\mathrm{mg} / \mathrm{dl})$ was determined by the glucose oxidase color method (Glucose LR, GOD-PAP; Linear Chemicals, Barcelona, Spain). Determinations of serum total cholesterol, high-density lipoprotein (HDL-C), and triglycerides were performed using the Siemens Advia 1650 Clinical Chemistry System (Siemens Medical Solutions, Erlangen, Germany). Cholesterol bound to low-density lipoprotein (LDL-C) was estimated by the Friedewald equation. Internal quality control of the lipids was carried out according to the laboratory manual of the Lipid Research Clinics Program. Insulin $(\mu \mathrm{U} / \mathrm{ml})$ was measured by a solidphase enzyme-amplified sensitivity immunoassay (INSEASIA; Biosource Technologies, Nivelles, Belgium). Total testosterone (ng/dl) was measured by ELISA (testosterone enzyme immunoassay test kit, LI7603; Linear Chemicals). SHBG (nmol/l) serum levels were measured by ELISA (SHBG ELISA, MX 520 11; IBL, Hamburg, Germany). Serum $E_{2}$ was measured by RIA (Immunometrics, London, UK). Plasma samples were analyzed for free testosterone $(\mathrm{pg} / \mathrm{ml})$ using the commercially available Coat-A-Count FT kit (Diagnostic Products, Los Angeles, CA, USA). DHEA-S (ng/ml) serum levels were measured by DSL DHEA-S RIA kit (Diagnostic Systems Laboratories, Webster, TX, USA). LH (IU/l), and FSH (IU/l) were measured using the LHsp and FSH IRMA kits from Biosource Technologies. $\Delta 4 \mathrm{~A}(\mathrm{ng} / \mathrm{ml})$ was measured by RIA using active androstenedione-coated tube RIA kit DSL 3800 (Diagnostic Systems Laboratories). 17-OHP was 
determined by RIA kit (ICN Pharmaceuticals Inc Costa Mesa, CA, USA). The intra- and inter-assay coefficients of variation $(\mathrm{CVs})$ were $<5.0 \%$ and $7.5 \%$ respectively for all the analytes.

Determination of hsCRP was performed by a fully mechanized latex particle-enhanced immunonephelometric assay on the BN ProSpec nephelometer (Dade Behring, Liederbach, Germany). The intra- and interassay CVs were $<6$ and $7 \%$ respectively. Serum NGAL and MMP-9/NGAL levels were determined by solid-phase ELISA techniques (R\&D Systems, Minneapolis, MN, USA). The intra- and inter-assay CVs for NGAL ranged between 3.1 and $4.1 \%$ and 5.6 and $7.9 \%$ respectively, and for MMP-9/NGAL between 2.3 and $4.1 \%$ and 5.1 and $7.6 \%$ respectively.

\section{Statistical analysis}

All continuous variables showed normal distribution as it was documented by the use of the KolmogorovSmirnov test. Data are presented as means and S.D. The two-tailed unpaired $t$-test was used to evaluate the differences in normally distributed variables between the two groups. The Pearson correlation coefficient was applied to assess the correlation between the variables. Statistical analysis was performed using the statistical package SPSS 13.00 version (Statistical Package for the Social Sciences, Chicago, IL, USA). Statistical significance was set at $P<0.05$.

\section{Results}

The two groups of patients and controls were matched for age $(25.4 \pm 4.5$ vs $27.4 \pm 4.4$ years, $P>0.05)$ and BMI $\left(25.37 \pm 3.8\right.$ vs $\left.23.46 \pm 2.4 \mathrm{~kg} / \mathrm{m}^{2}, P>0.09\right)$ respectively. NGAL and MMP-9/NGAL complex concentrations were significantly lower in PCOS group compared with controls $(30.4 \pm 24.3$ vs $70.7 \pm 37.9 \mu \mathrm{g} / \mathrm{l}, P<0.0001)$ and $(31.5 \pm 26.6$ vs $115.1 \pm 66.9 \mu \mathrm{g} / \mathrm{l}, \quad P<0.0001)$ respectively. Androgen values, namely total testosterone $(0.83 \pm 0.21$ vs $0.48 \pm 0.18 \mathrm{ng} / \mathrm{dl}, P<0.0001)$, free testosterone $(2.45 \pm 0.69$ vs $1.67 \pm 0.51 \mathrm{pg} / \mathrm{ml}$, $P<0.0001), \Delta 4 \mathrm{~A} \quad(3.0 \pm 1.21$ vs $2.2 \pm 0.73 \mathrm{ng} / \mathrm{ml}$, $P<0.002)$, and DHEAS $(250 \pm 20.89$ vs $185.61 \pm$ $13.75 \mathrm{ng} / \mathrm{ml}, P<0.01)$ were significantly higher in PCOS subjects. Lipids, $E_{2}$ levels $(57.6 \pm 7$ vs $62.31 \pm$ $10.29 \mathrm{pmol} / \mathrm{l}, P>0.06)$, and LH/FSH ratio ( $1.2 \pm 0.14 \mathrm{vs}$ $0.84 \pm 0.14, P>0.64)$ were comparable among the two groups. Insulin resistance index HOMA-IR $(2.31 \pm 1.01$ vs $1.8 \pm 0.96, P<0.02)$ and $\operatorname{hsCRP}(3.8 \pm 0.92$ vs $1.78 \pm$ $0.3 \mathrm{mg} / \mathrm{l}, P<0.05)$ were significantly higher in PCOS than in controls (Table 1 ).

When NGAL and MMP-9/NGAL levels were stratified according to BMI status in patients and controls, namely lean with BMI $\leq 25 \mathrm{~kg} / \mathrm{m}^{2}$ and overweight with BMI $>25 \mathrm{~kg} / \mathrm{m}^{2}$, we found that NGAL levels were significantly lower in either lean $(n=25 ; 31.9 \pm 5.3$
Table 1 Pertinent data in polycystic ovary syndrome (PCOS) subjects and controls.

\begin{tabular}{|c|c|c|c|}
\hline & $\begin{array}{l}\text { PCOS } \\
(n=40)\end{array}$ & $\begin{array}{c}\text { Controls } \\
(n=40)\end{array}$ & $P$ value \\
\hline Age (years) & $25.4 \pm 4.5$ & $27.4 \pm 4.4$ & 0.06 \\
\hline BMI $\left(\mathrm{kg} / \mathrm{m}^{2}\right)$ & $25.3 \pm 3.8$ & $23.4 \pm 2.4$ & 0.09 \\
\hline Total cholesterol $(\mathrm{mg} / \mathrm{dl})$ & $184.8 \pm 31.6$ & $169.4 \pm 33.7$ & 0.12 \\
\hline Triglycerides (mg/dl) & $90.5 \pm 44.6$ & $89.2 \pm 56.6$ & 0.93 \\
\hline $\mathrm{HDL}-\mathrm{C}(\mathrm{mg} / \mathrm{dl})$ & $51.9 \pm 9.5$ & $49.0 \pm 9.9$ & 0.33 \\
\hline LDL-C (mg/dl) & $114.8 \pm 29.6$ & $100.6 \pm 26$ & 0.11 \\
\hline LH/FSH & $1.2 \pm 0.1$ & $0.8 \pm 0.1$ & 0.08 \\
\hline $\mathrm{E}_{2}(\mathrm{pmol} / \mathrm{l})$ & $57.6 \pm 7$ & $62.3 \pm 10.3$ & 0.07 \\
\hline TESTO (ng/dl) & $0.8 \pm 0.21$ & $0.4 \pm 0.1$ & 0.0001 \\
\hline SHBG $(\mathrm{nmol} / \mathrm{l})$ & $38.8 \pm 17.3$ & $50.5 \pm 21.9$ & 0.01 \\
\hline FTESTO (pg/ml) & $2.4 \pm 0.7$ & $1.67 \pm 0.51$ & 0.0001 \\
\hline 17-OHP (ng/ml) & $1.2 \pm 0.6$ & $0.9 \pm 0.3$ & 0.06 \\
\hline$\Delta 4 \mathrm{~A}(\mathrm{ng} / \mathrm{ml})$ & $3 \pm 1.2$ & $2.2 \pm 0.7$ & 0.002 \\
\hline DHEAS (ng/ml) & $250 \pm 21$ & $185 \pm 14$ & 0.01 \\
\hline HOMA-IR & $2.3 \pm 1$ & $1.8 \pm 0.9$ & 0.02 \\
\hline hsCRP (mg/l) & $3.8 \pm 0.9$ & $1.8 \pm 0.3$ & 0.05 \\
\hline NGAL $(\mu \mathrm{g} / \mathrm{l})$ & $30.4 \pm 24.3$ & $70.7 \pm 38$ & 0.0001 \\
\hline MMP-9/NGAL $(\mu \mathrm{g} / \mathrm{l})$ & $31.6 \pm 26.6$ & $115.1 \pm 66.9$ & 0.0001 \\
\hline
\end{tabular}

S.D. is presented for age, BMI, and lipids. In all other variables, the S.E.M. is presented.

vs $70.7 \pm 7.5 \mu \mathrm{g} / \mathrm{l}, P<0.0002)$ or overweight $(n=15$; $26.7 \pm 5.6$ vs $72.5 \pm 10.9, P<0.0004)$ PCOS patients compared with controls (lean, $n=30$; overweight, $n=10)$. Also, MMP-9/NGAL levels were significantly lower in lean $(29.0 \pm 6.6$ vs $119.5 \pm 12.8 \mu \mathrm{g} / \mathrm{l}$, $P<0.0001)$ and overweight $(37.6 \pm 11.3$ vs $110.7 \pm 18.9, P<0.002)$, PCOS subjects compared with controls (Fig. 1A and B). The two subgroups, lean and obese/overweight, when divided to PCOS patients and controls, have similar differences among them. Namely, they did not differ in age $(24 \pm 0.8$ vs $25.93 \pm$ 0.84 and $25.00 \pm 1.81$ vs $28.13 \pm 1.1$ years, in lean and overweight respectively). Also, the two subgroups were comparable for BMI $(21.8 \pm 0.4$ vs $21.3 \pm 0.4$ and $31.3 \pm 1.4$ vs $29.3 \pm 1.5 \mathrm{~kg} / \mathrm{m}^{2}$ in lean and overweight respectively). Additionally, $\mathrm{E}_{2}$ values were similar in the subgroups $(60.5 \pm 14.7$ vs $51.14 \pm 6.0$ and $57.3 \pm 15.3$ vs $53.97 \pm 11.64 \mathrm{pmol} / \mathrm{l})$. Testosterone values were significantly higher in PCOS group in either lean $(0.48 \pm 0.02$ vs $0.81 \pm 0.05 \mathrm{ng} / \mathrm{dl}, P<0.0001)$ or overweight $(0.81 \pm 0.05$ vs $0.48 \pm 0.02 \mathrm{ng} / \mathrm{dl}, P<0.0001)$. Based on HOMA-IR index, PCOS overweight subgroups were more insulin resistant than controls $(2.64 \pm 0.33$ vs $1.7 \pm 0.17, P=0.01)$, whereas lean PCOS were comparable with controls $(2.03 \pm 0.14$ vs $1.65 \pm 0.18$, $P=$ NS). Finally, hsCRP values were higher in PCOS than in controls in both subgroups, but this difference did not reach statistical significance in either subgroup $(2.47 \pm$ $0.77 \mathrm{vs} 1.2 \pm 0.29 \mathrm{mg} / \mathrm{l}$ and $2.47 \pm 0.77$ vs $1.2 \pm 0.29$, in lean and overweight respectively).

Considering the total number of subjects, PCOS and controls, NGAL levels were positively correlated with age $(r=0.337, P<0.002)$ and negatively with testosterone $(r=-0.348, P<0.002)$, free testosterone $(r=-0.325$, 

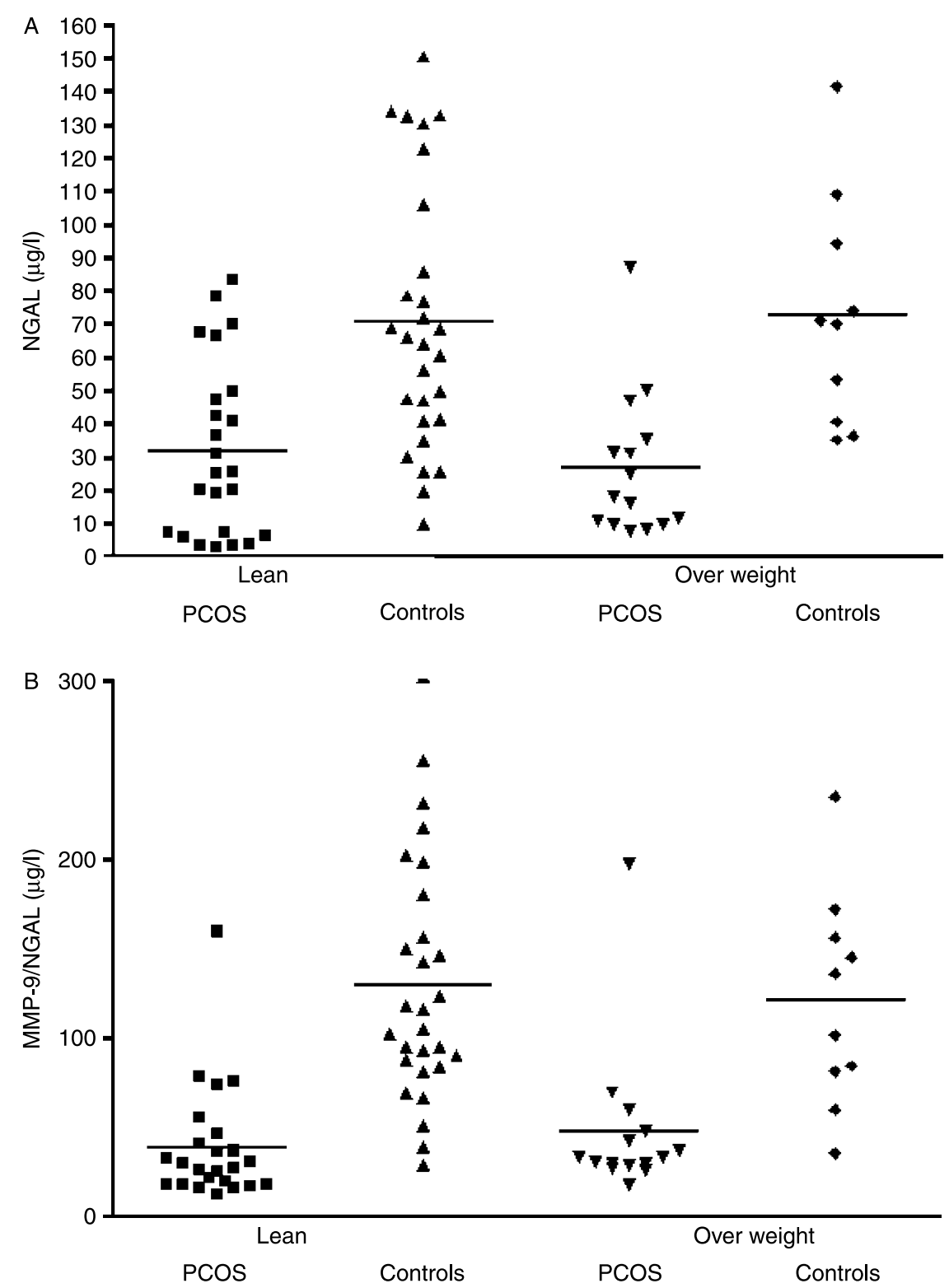

Figure 1 (A) NGAL levels in lean and overweight PCOS and controls (unpaired $t$-test). (B) NGAL/MMP-9 NGAL levels in lean and overweight $\mathrm{PCOS}$ and controls (unpaired $t$-test). Lean, $\mathrm{BMI} \leq 25$; overweight, $\mathrm{BMI}>25 \mathrm{~kg} / \mathrm{m}^{2}$.

$P<0.01)$, and DHEAS values $(r=-0.263, P<0.004)$. MMP-9/NGAL complex was also positively correlated with age $(r=0.451, \quad P<0.001)$ and negatively correlated with testosterone $(r=-0.457, P<0.001)$, free testosterone $(r=-0.394, \quad P<0.002), \quad \Delta 4 \mathrm{~A}$ $(r=-0.313, P<0.02)$, DHEAS values $(r=-0.324$, $P<0.009)$, and glucose values $(r=-0.227, P<0.05)$. Neither NGAL nor MMP-9/NGAL complex were correlated with BMI, lipids, gonadotropins, $\mathrm{E}_{2}, 17-\mathrm{OHP}$, SHBG, insulin, HOMA-IR, and hsCRP.

When PCOS and controls were studied as separate groups, a negative association was observed between NGAL and MMP-9/NGAL complex with testosterone, free testosterone, and DHEAS in PCOS, whereas age was positively correlated with both NGAL and MMP-9/NGAL complex in both groups (Table 2).

\section{Discussion}

In this study, it was demonstrated that serum concentrations of NGAL and MMP-9/NGAL, two welldefined factors in the evolution of atherogenic processes, were significantly lower in PCOS than those in control women $(8,14)$. These findings suggest that the 


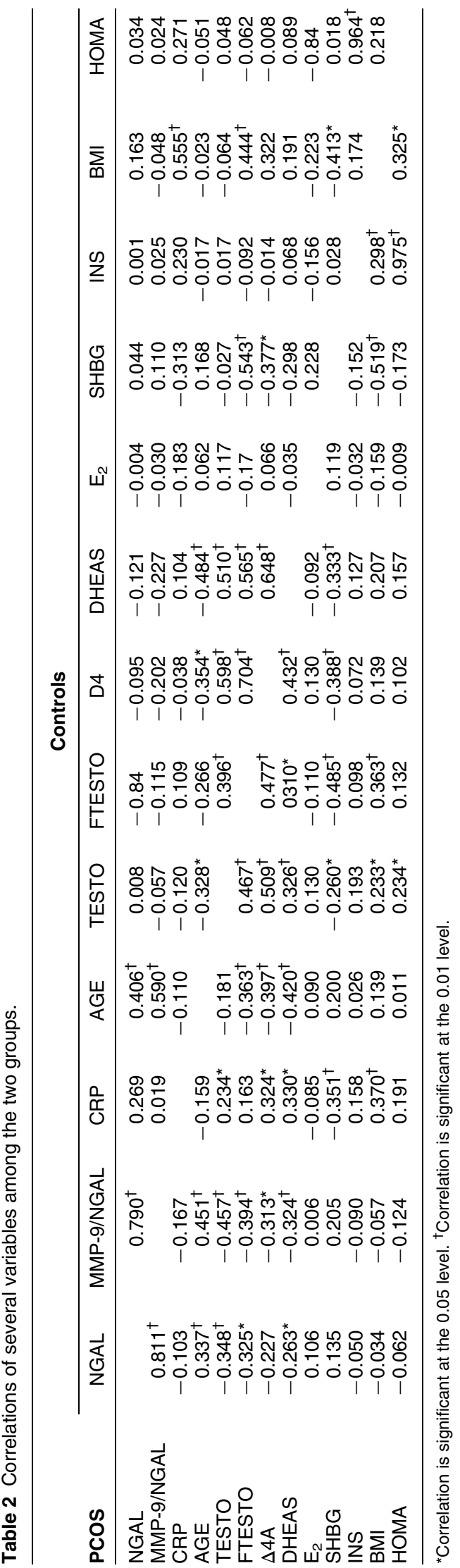

decreased levels of disruptors of atherosclerotic plaque may protect PCOS subjects from manifesting cardiovascular events, despite the presence of increased risk factors (20). In several studies concerning patients with PCOS, endothelial dysfunction, fibrinolytic derangements, insulin resistance, and subclinical inflammation have been detected, suggesting that these patients are at high risk of developing cardiovascular incidents (21, 22). The fact that in PCOS patients overt cardiovascular incidents are not frequently encountered indicates possible protective factors in PCOS subjects that delay or inhibit atherogenic process.

MMP-9 is a proteolytic enzyme participating in the remodeling of extracellular matrix. An increased activity of MMP-9 has been reported in atherosclerosis and cardiovascular disease (23). NGAL or lipocalin 2 is thought to be an acute phase protein whose expression is up-regulated in humans, typically in epithelial cells, under diverse inflammatory conditions (24). Additionally, a role of NGAL as a mediator of insulin resistance has been recently suggested $(25,26)$. Overall, the full functional repertoire of NGAL remains to be elucidated. Of particular interest is the finding that NGAL inhibits MMP-9 inactivation, leading to enhanced proteolytic activity with prolonged effects on collagen degradation (27). Indeed, Hemdal et al. have found increased expression and co-localization of MMP-9 and NGAL in atherosclerotic lesions, especially in myocardial infarction (15).

Considering the fact that NGAL and MMP-9 are clearly involved in the final steps of vascular damage, their decreased concentrations in PCOS subjects indicate that they might be part of a protective mechanism. In the present study, the significantly lower concentrations of NGAL and MMP-9/NGAL complex appear to be independent of the degree of obesity. Low concentrations of NGAL and MMP9/NGAL complex cannot be attributed to estrogen levels, since they were similar among patients and controls. The negative association of these atherogenic molecules with androgens advocates a probable role of androgens in cardiovascular protection in PCOS subjects. The role of androgens in cardiovascular risk factors in women with PCOS has been controversial; however, there are studies suggesting a protective role of androgens $(28,29)$. Interestingly, no association of NGAL and MMP-9/NGAL complex with insulin resistance or hsCRP was demonstrated in this study, a finding that implies alternative pathways of NGAL and MMP-9 regulation.

To our knowledge, this is the first study addressing NGAL and MMP-9/NGAL complex in PCOS subjects. Lewandowski et al. have studied MMP-9 concentration in PCOS and they have detected elevated values in PCOS women (30). However, their studied group of PCOS consisted of significantly heavier and older women than in the present study (BMI $35.8 \pm 7.5$ vs $25.4 \pm 3.8 \mathrm{~kg} / \mathrm{m}^{2}$, age $30.5 \pm 6.7$ vs $25.4 \pm 4.5$ years). 
These parameters affect the final outcome since in both studies, MMP-9 and NGAL/MMP-9 complex were positively related to age, and in the study of Lewandowski et al. there was a positive association between MMP-9 and BMI. Noticeably, NGAL and MMP-9/NGAL complex is a more sensitive marker of atherogenic risk in humans than solely MMP-9, since these two factors taken together act in cooperation leading to plaque erosion and consequently the evolution of cardiovascular events.

In conclusion, the presented data indicate that NGAL and MMP-9/NGAL complex, two molecules activating the erosion of atherotic plaque, are lower in PCOS subjects. These findings could contribute in elucidating the discrepancy between the increased estimated prevalence as opposed to lower clinically confirmed incidence of cardiovascular events in women with PCOS.

\section{Acknowledgements}

Grant/funding support: funding was received from the Athens University Medical School (to E D-K). The funding source played no role in the study design; in the collection, analysis, and interpretation of data; in the writing of the report; or in the decision to submit the report for publication.

\section{References}

1 Diamanti-Kandarakis E, Kouli CR, Bergiele AT, Filandra FA, Tsianateli TC, Spina GG, Zapanti ED \& Bartzis MI. A survey of the polycystic ovary syndrome in the Greek island of Lesbos: hormonal and metabolic profile. Journal of Clinical Endocrinology and Metabolism $1999 \mathbf{8 4} 4006-4011$.

2 Dunaif A. Insulin resistance and the polycystic ovary syndrome: mechanism and implications for pathogenesis. Endocrine Reviews $199718774-800$.

3 Ehrmann DA, Liljenquist DR, Kasza K, Azziz R, Legro RS \& Ghazzi MN. Prevalence and predictors of the metabolic syndrome in women with polycystic ovary syndrome. Journal of Clinical Endocrinology and Metabolism 20069 48-53.

4 Diamanti-Kandarakis E, Alexandraki K, Protogerou A, Piperi C, Papamichael C, Aessopos A, Lekakis J \& Mavrikakis M. Metformin administration improves endothelial function in women with polycystic ovary syndrome. European Journal of Endocrinology 2005 152 749-756.

5 Orio F, Palomba S, Cascella T, De Simone B, Di Biase S, Russo T, Labella D, Zullo F, Lombardi G \& Colao A. Early impairment of endothelial structure and function in young normal-weight women with polycystic ovary syndrome. Journal of Clinical Endocrinology and Metabolism $2004894588-4593$.

6 Talbott EO, Zborowski JV, Rager JR, Boudreaux MY, Edmundowicz DA \& Guzick DS. Evidence for an association between metabolic cardiovascular syndrome and coronary and aortic calcification among women with polycystic ovary syndrome. Journal of Clinical Endocrinology and Metabolism 2004 89 5454-5461.

7 Coviello AD, Legro RS \& Dunaif A. Adolescent girls with polycystic ovary syndrome have an increased risk of the metabolic syndrome associated with increasing androgen levels independent of obesity and insulin resistance. Journal of Clinical Endocrinology and Metabolism $200691492-497$.
8 Galis ZS, Sukhova GK, Lark MV \& Libby P. Increased expression of matrix metalloproteinases and matrix degrading activity in vulnerable regions of human atherosclerotic plaques. Journal of Clinical Investigation 199494 2493-2503.

9 Kai H, Ikeda H, Yasukawa H, Kai M, Seki Y, Kuwahara F, Ueno T, Sugi K \& Imaizumi T. Peripheral blood levels of matrix metalloproteinase-2 and -9 are elevated in patients with acute coronary syndromes. Journal of the American College of Cardiology 199832 368-372.

10 Tayebjee MH, Lip GY \& MacFadyen RJ. Matrix metalloproteinases in coronary artery disease: clinical and therapeutic implications and pathological significance. Current Medicinal Chemistry 2005 12 917-925.

11 Tong Z, Wu X, Ovcharenko D, Zhu J, Chen CS \& Kehrer JP. Neutrophil gelatinase-associated lipocalin as a survival factor. Biochemical Journal $2005391441-448$.

12 Leclercq A, Houard X, Philippe M, Ollivier V, Sebbag U, Meilhac O \& Michel JB. Involvement of intraplaque hemorrhage in atherothrombosis evolution via neutrophil protease enrichment. Journal of Leukocyte Biology 200782 1420-1429.

13 Yan L, Borregaard N, Kjeldsen L \& Moses MA. The high molecular weight urinary matrix metalloproteinase (MMP) activity is a complex of gelatinase B/MMP-9 and neutrophil gelatinaseassociated lipocalin (NGAL). Journal of Biological Chemistry 2001 $27637258-37265$.

14 Bu DX, Hemdahl AL, Gabrielsen A, Fuxe J, Zhu C, Eriksson P \& Yan Z. Induction of neutrophil gelatinase-associated lipocalin in vascular injury via activation of nuclear factor-B. American Journal of Pathology $20061692245-2253$.

15 Hemdahl AL, Gabrielsen A, Zhu C, Eriksson P, Hedin U, Kastrup J, Thoren P \& Hansson GK. Expression of neutrophil gelatinase - associated lipocalin in atherosclerosis and myocardial infarction. Arteriosclerosis, Thrombosis, and Vascular Biology 200626 136-142.

16 Lo JC, Feigenbaum SL, Yang J, Pressman AR, Selby JV \& Go AS. Epidemiology and adverse cardiovascular risk profile of diagnosed polycystic ovary syndrome. Journal of Clinical Endocrinology and Metabolism 200691 1357-1363.

17 Wild S, Pierpoint T, McKeigue P \& Jacobs H. Cardiovascular disease in women with polycystic ovary syndrome at long-term follow-up: a retrospective cohort study. Clinical Endocrinology 2000 52 595-600.

18 National Institutes of Health Consensus Meeting on PCOS. In Current Issues in Endocrinology and Metabolism, Ed. A Dunaif, Boston: Blackwell Scientific, 1992.

19 Matthews DR, Hosker JP, Rudenski AS, Naylor BA, Treacher DF \& Turner RC. Homeostasis model assessment: insulin resistance and beta-cell function from fasting plasma glucose and insulin concentrations in man. Diabetologia 1985 28 412-419.

20 Cussons AJ, Stuckey BG \& Watts GF. Cardiovascular disease in the polycystic ovary syndrome: new insights and perspectives. Atherosclerosis $2006185227-239$.

21 Glueck CJ, Papanna P, Wang P, Gildenberg N \& Sieve-Smith L. Incidence and treatment of metabolic syndrome in newly referred women with confirmed polycystic ovarian syndrome. Metabolism 200352 908-915.

22 Diamanti-Kandarakis E, Paterakis T, Alexandraki K, Piperi C, Aessopos A, Katsikis I, Katsilambros G \& Panidis D. Indices of lowgrade chronic inflammation in polycystic ovary syndrome and the beneficial effect of metformin. Human Reproduction $2006 \mathbf{2 1}$ 1426-1431.

23 Newby AC. Dual role of matrix metalloproteinases (matrixins) in intimal thickening and atherosclerotic plaque rupture. Physiological Reviews 200585 1-31.

24 Flower DR. The lipocalin protein family: structure and function. Biochemical Journal $19963181-14$.

25 Yan QW, Yang Q, Mody N, Graham TE, Hsu CH, Xu Z, Houstis NE, Kahn BB \& Rosen ED. The adipokine lipocalin 2 is regulated by obesity and promotes insulin resistance. Diabetes $2007 \mathbf{5 6}$ 2533-2540. 
26 Wang Y, Lam KS, Kraegen EW, Sweeney G, Zhang J, Tso AW, Chow WS, Wat N, Xu JY, Hoo R \& Xu A. Lipocalin-2 is an inflammatory marker closely associated with obesity, insulin resistance, and hyperglycemia in humans. Clinical Chemistry 200753 134-141.

27 Loftus IM, Naylor AR, Goodall S, Crowther M, Jones L, Bell PR \& Thompson MM. Increased matrix metalloproteinase-9 activity in unstable carotid plaques. A potential role in acute plaque disruption. Stroke 200031 40-47.

28 Liu P, Death A \& Handelsman D. Androgens and cardiovascular disease. Endocrine Reviews 200324 313-340.

29 Bernini GP, Sagro M, Moretti A, Argenio GF, Barlascini CO, Cristofani R \& Salvetti A. Endogenous androgens and carotid intimal-medial thickness in women. Journal of Clinical Endocrinology and Metabolism 199984 2008-2012.

30 Lewandowski KC, Komorowski J, O'Callaghan CJ, Tan BK, Chen J, Prelevic GM \& Randeva HS. Increased circulating levels of matrix metalloproteinase-2 and -9 in women with the polycystic ovary syndrome. Journal of Clinical Endocrinology and Metabolism 2006 91 1173-1177.

Received 21 December 2007

Accepted 2 January 2008 